Konrad-Zuse-Zentrum

für Informationstechnik Berlin

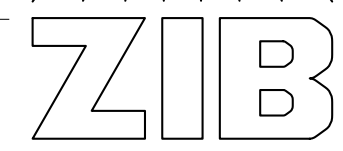

Marcus Weber, Susanna Kube, Lionel Walter, Peter Deuflhard

\title{
Stable computation of probability densities for metastable dynamical systems
}





\title{
Stable computation of probability densities for metastable dynamical systems*
}

\author{
Marcus Weber, Susanna Kube, Lionel Walter, Peter Deuflhard
}

November 15, 2006

\begin{abstract}
Whenever the invariant stationary density of metastable dynamical systems decomposes into almost invariant partial densities, its computation as eigenvector of some transition probability matrix is an ill-conditioned problem. In order to avoid this computational difficulty, we suggest to apply an aggregation/disaggregation method which only addresses wellconditioned sub-problems and thus results in a stable algorithm. In contrast to existing methods, the aggregation step is done via a sampling algorithm which covers only small patches of the sampling space. Finally, the theoretical analysis is illustrated by two biomolecular examples.
\end{abstract}

AMS MSC 2000: 65F15, 82B80, 62H30

Keywords: dynamical systems, metastability, molecular conformations, cluster analysis, sampling, aggregation/disaggregation, domain decomposition

*Supported by the DFG Research Center Matheon "Mathematics for key technologies" in Berlin 


\section{Contents}

Introduction 3

1 Difficulties of existing sampling methods 4

1.1 Conformation dynamics revisited . . . . . . . . . . . . 4

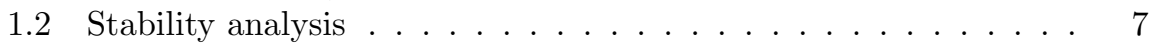

1.3 An illustrative example . . . . . . . . . . . . . . 9 9

2 Sampling based aggregation/disaggregation $\quad 12$

2.1 Basic idea . . . . . . . . . . . . . . . . . . . . . . . . 12

2.2 Global aggregation . . . . . . . . . . . . . . . . 14

2.3 Local aggregation via density estimation . . . . . . . . . . . 16

2.4 Numerical Examples . . . . . . . . . . . . . . . . . . . . . . 17

$\begin{array}{ll}\text { Conclusion } & 21\end{array}$ 


\section{Introduction}

Consider general dynamical systems in equilibrium with a unique stationary density. Among them, metastable dynamical systems are characterized by the fact that there exist almost invariant subsets wherein the system remains for "a long time", once it is in there. In molecular dynamical systems, which represent the main class of interest here, the almost invariant subsets are the metastable conformations; for general dynamical systems, we also speak of metastable clusters. Throughout this paper, we fix the stationary density to be the Boltzmann distribution without loss of generality. Within each of the conformations, the dynamics is often said to be "rapidly mixing", whereas between them it is "slowly mixing". Any of the available sampling strategies will, when applied to such systems, suffer from the undesirable fact that they are prone to be "trapped" within conformations. Difficulties of this kind also arise in a special multi-scale approach called conformation dynamics, which has been developed in recent years (for surveys see $[10,35,11]$ ) and which will represent our algorithmic frame here.

In this paper, we deal with the trapping problem as it shows up in computational sampling techniques for metastable dynamical systems. We design an efficient sampling strategy, especially for high-dimensional systems, which both exploits the fast mixing within the conformations and, at the same time, safely covers the rare transitions between the conformations. For our computations, we use the meshless implementation ZIBgridfree [45, 44] of conformation dynamics. In the presence of metastabilities, the arising Markov chains will be nearly completely decomposable or nearly reducible [39, 7, 29, 8]. The metastable conformations to be identified are analyzed via a stochastic transfer matrix, using our recently developed Robust Perron Cluster Analysis (PCCA+) [12, 44]. The basic reasoning behind the approach to be advocated herein is the following: As we will show below, the computation of the stationary density via the transfer matrix is highly ill-conditioned, whereas the identification of the metastable conformations is well-conditioned. On the basis of this insight, we suggest a domain decomposition of the state space into sub-domains, ideally the conformations or certain computationally available approximations of them. Our method may be regarded as an infinite dimensional analog of aggregation/disaggregation methods known from finite dimensional problems. The disaggregation step comprises the determination of the partial Boltzmann densities, using our algorithm ZIBgridfree. The aggregation step realizes the construction of the overall stationary density, using our newly developed algorithm ConfJump, which works efficiently only when applied to the already identified conformations.

The paper is organized as follows. Existing sampling algorithms and their difficulties in the treatment of metastable dynamical systems are analyzed in Section 1. In Section 2, we derive the basic idea of aggregation/disaggregation in function space and show how our proposed method can overcome the described difficulties. Finally, we present three numerical examples, one trivial but elucidating, the other two from biochemistry. 


\section{Difficulties of existing sampling methods}

In recent years, the efficient exploration of molecular state spaces and the identification of metastable conformations have been in the focus of our research, for surveys see $[10,11,35,36]$. Our algorithms are embedded in the mixed deterministic/stochastic approach called conformation dynamics. This approach is based on the analysis of a stochastic transfer operator corresponding to a specific Markov chain and, after some spatial discretization, on a Perron cluster analysis of a stochastic matrix. In the present section, we will shortly revisit conformation dynamics algorithms as they have been used up to now. In the sampling part of these algorithms, some computational dilemma arises unavoidably, as we will show. However, the same kind of difficulty would equally arise with any other sampling technique when applied to metastable dynamical systems.

\subsection{Conformation dynamics revisited}

Throughout the paper, we consider a canonical ensemble where the stationary density of states $q$ in a continuous state space $\Omega$ is given by the Boltzmann density $\pi(q)$. Conformation dynamics aims at a description of the continuous dynamical process in terms of a jump process on the discrete set of conformations. Given a molecule's geometry, we identify $n_{C}$ metastable conformations $C_{1}, \ldots, C_{n_{C}} \subset \Omega$ together with their life times and transition patterns. Conformations are given by measurable functions $\xi_{i}$ defined in $\Omega$ (e.g. characteristic functions of the sets $\left\{C_{i}\right\}$ ), which, together with the Boltzmann density, determine the conformational weights or, more general, cluster weights

$$
\sigma_{i}^{\text {exact }}=\int_{\Omega} \xi_{i}(q) \pi(q) d q, \quad i=1, \ldots, n_{C} .
$$

In order to make the computations feasible, the continuous state space is decomposed into a number of $\mathrm{N}$ discrete "spatial" states, $E=\{1, \ldots, N\}$, and the discrete dynamical process is described as a Markov chain on these states. The stationary density of the corresponding transition probability matrix is denoted by $\mathbf{w}=\left(w_{1}, \ldots, w_{N}\right)$, in contrast to the stationary density $\pi(q)$ of the continuous process. The entry $w_{i}$ of the discrete stationary density is denoted as statistical weight of state $i \in E$. The vector $\mathbf{w}$ arises as normalized left eigenvector of the transition probability matrix $P$, i.e.

$$
\mathbf{w}^{\top} P=\mathbf{w}^{\top} .
$$

As a result of our cluster algorithm PCCA + , membership vectors $\left\{\chi_{i}\right\}_{i=1}^{N}$ are obtained, in contrast to the continuous functions $\xi_{i}(q)$. The entry $\chi_{i}(j)$ is a number between 0 and 1 and denotes the grade of membership of state $j$ w.r.t. conformation $i$. Given these values and the stationary density $\mathbf{w}$, the continuous cluster weights in (1) are approximated by discrete cluster weights

$$
\sigma_{i}=\sum_{j=1}^{N} \chi_{i}(j) w_{j} \approx \sigma_{i}^{\text {exact }} .
$$

The information about the discrete cluster dynamics is condensed in the so-called coupling matrix $\mathcal{P}$, the stochastic matrix describing the transitions between the metastable conformations. With diagonal matrices $D=\operatorname{diag}\left(\left\{w_{i}\right\}_{i=1}^{n_{C}}\right)$ 
and $\widetilde{D}=\operatorname{diag}\left(\left\{\sigma_{i}\right\}_{i=1}^{n_{C}}\right)$, this matrix can be computed via

$$
\mathcal{P}=\widetilde{D}^{-1} \chi^{\top} D P \chi .
$$

From [10], the characteristic life times of conformations are known to behave (in first order) like

$$
\tau_{i} \doteq \frac{\tau}{1-p_{i i}},
$$

where $\tau$ is the molecular dynamics simulation time step. Note that these times are extremely sensitive to perturbations of $p_{i i}<1$ when $p_{i i} \approx 1$.

Summarizing, both the computation of the relevant dynamical information $\mathcal{P}$ and the cluster weights $\left\{\sigma_{i}\right\}$ depend on a well-conditioned determination of $\mathbf{w}$ in (2) and a reasonable clustering $\chi$.

Sampling strategy. Within conformation dynamics, a Hybrid Monte Carlo (HMC) sampling technique [13] is used, which is known as a quite efficient method for the sampling of canonical ensembles. However, as with other sampling strategies, its main problem is the so-called "trapping" effect, which means that the sampling remains for a "too long" time in the neighborhood of some potential well, an effect also named as "critical slowing down". As a consequence, the method fails to visit further physically relevant parts of the conformational space - a significant lack of reliability. One possibility to circumvent this undesirable effect is to modify the potential energy surface accordingly and to re-weight the generated sampling points. However, such techniques require some deep knowledge about the energy landscape and tend to accumulate too many low-weighted sampling points in transition regions. Therefore, we will advocate below the use of a domain decomposition method instead. Its realization requires our recently developed software package ZIBgridfree to be presented next.

Meshless algorithm ZIBgridfree[45, 44]. The name of this algorithm stands for a gridfree (or meshless) partitioning of the sampling space such that different regions can be sampled independently. The decomposition of high-dimensional spaces is a difficult problem of its own. Regular grids are well-known to be inappropriate, because they suffer from the "curse of dimensionality", for an illustration see Figure 1, left, where the line represents the conformation domain. In order to overcome this problem, this algorithm realizes a decomposition into "domains" $\left\{\Omega_{i}\right\}$ via overlapping sigmoidal functions $\left\{\phi_{i}\right\}$, see Figure 1, right. Thus, more precisely, the algorithm realizes a decomposition in function space rather than in position space. However, even though the support of these basis functions is the whole state space $\Omega$, they are strongly localized in the conformation domain. Hence, we may as well loosely speak of domain decomposition in $\Omega$. Throughout the paper, we assume that we have already realized some decomposition by means of $N$ basis functions $\phi_{1}, \ldots, \phi_{N}: \Omega \rightarrow[0,1]$, where $\phi_{i}(q)$ determines the grade of membership of position state $q \in \Omega$ w.r.t. $\Omega_{i}$. For later purposes, we already mention here that this algorithm may be regarded as realizing a disaggregation step in infinite dimension.

In detail, the dynamics is described via a Markov operator $T^{\tau}$ [35] that propagates densities in $\Omega$ for some time $\tau$. The corresponding transition probabilities between domains $\Omega_{i}$ can be expressed in terms of a stochastic transition 

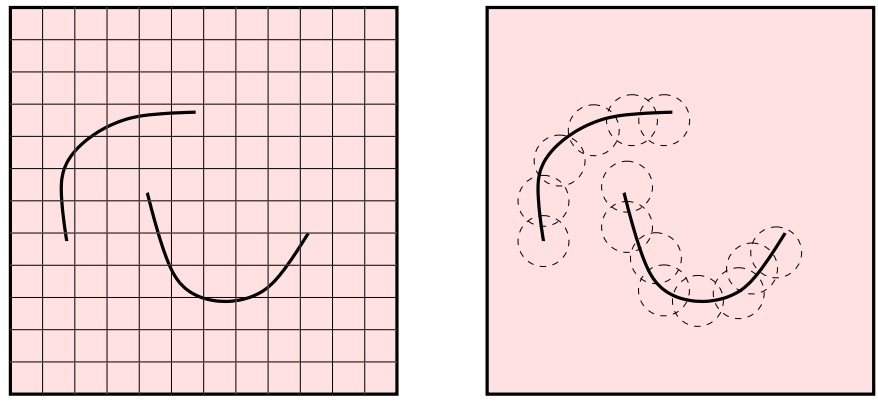

Figure 1: Different discretizations of conformations (lines) in position space. Left: Regular grids require too many unnecessary boxes, giving rise to the "curse of dimensionality". Right: Meshless methods realize an adaptive, functionbased and overlapping discretization. The circles indicate the "domains" $\left\{\Omega_{i}\right\}$ corresponding to the strongly localized Gaussian radial basis functions $\left\{\phi_{i}\right\}$.

matrix $P \in \mathbb{R}^{N \times N}$ as follows,

$$
P(i, j)=\frac{\int_{\Omega} \phi_{i}(q) T^{\tau} \phi_{j}(q) \pi(q) d q}{\int_{\Omega} \phi_{i}(q) \pi(q) d q} .
$$

It can easily be shown that the integral can be rewritten in terms of local densities

$$
\pi_{i}(q)=\frac{\phi_{i}(q) \pi(q)}{\int_{\Omega} \phi_{i}(q) \pi(q)}
$$

as

$$
P(i, j)=\int_{\Omega} T^{\tau} \phi_{j}(q) \pi_{i}(q) d q .
$$

The local densities are Boltzmann densities corresponding to modified potentials $\left\{V_{i}(q)\right\}$, which depend on the basis functions $\left\{\phi_{i}(q)\right\}$, so that

$$
\pi_{i}(q)=\frac{\exp \left(-\beta V_{i}(q)\right)}{\int_{\Omega} \exp \left(-\beta V_{i}(\bar{q})\right) d \bar{q}}
$$

with

$$
V_{i}(q)=V(q)-\frac{1}{\beta} \log \left(\phi_{i}(q)\right) .
$$

The first step of the sampling routine comprises the generation of sampling points $\left\{q_{k}^{(i)}\right\}_{k=1}^{M}$ according to the local density $\pi_{i}(q)$. This is similar to the famous umbrella sampling technique introduced by Torrie and Valleau [42]. The second step includes the application of a propagator $T^{\tau}$ to these sampling points and the evaluation of the integral by Monte Carlo quadrature,

$$
P(i, j) \approx \frac{1}{M} \sum_{k=1}^{M} \phi_{j}\left(T^{\tau} q_{k}^{(i)}\right) .
$$

Actually we compute an approximation of $P$, because Monte Carlo integration introduces truncation errors, which, within ZIBgridfree, are controlled by means of certain stopping criteria. For more details see [44]. 
Robust Perron Cluster Analysis (PCCA+). Transition matrices of completely decoupled Markov chains with $n_{C}$ independent clusters can be reordered to block diagonal structure and have an $n_{C}$-fold Perron eigenvalue $\left\{\lambda_{i}\right\}_{i=1}^{n_{C}}=1$. In the presence of metastabilities, we deal with nearly completely decomposable Markov chains. In this case, there exists a Perron cluster of eigenvalues near the Perron eigenvalue $\lambda_{1}=1$. The perturbation is measured as the gap to the 2nd largest eigenvalue [12], i. e. as

$$
\varepsilon=1-\lambda_{2}
$$

which is understood to be a small number, positive by construction.

In contrast to a reordering of states according to clusters, PCCA + aims at an assignment of states $i \in\{1, \ldots, N\}$ to clusters $j \in\left\{1, \ldots, n_{C}\right\}$ with certain grades of membership $\chi_{j}(i)$. Let $X \in \mathbb{R}^{N \times n_{C}}$ denote the matrix of the eigenvectors corresponding to the Perron eigenvalue cluster. In $[12,44]$ it has been shown that $\chi$ can be expressed as a linear combination of the eigenvectors

$$
\chi=X \mathcal{A},
$$

where $\mathcal{A} \in \mathbb{R}^{n_{C} \times n_{C}}$ is a regular matrix. The algorithm PCCA + computes this transformation matrix via maximizing the metastability, which is the sum of the diagonal entries of the coupling matrix.

Sampling algorithm ConfJump. In [43], we described the sampling algorithm ConfJump that uses the results of an a priori analysis of the potential energy surface to enhance the sampling by additional intermediate jumps between lowenergy regions. This method allows a much faster sampling than the hybrid Monte Carlo algorithm. There is a class of alternative methods, so-called smart methods, which have been designed to overcome the trapping problem, see [4] for a survey. ConfJump can be regarded as an extension of the Smart Darting Monte Carlo [2] and the Jump Between Wells (JBW) [37, 38] method. However, ConfJump achieves larger acceptance ratios than these methods, i.e., within the Monte-Carlo setting the part of proposed states which are accepted as next state in the Markov chain is larger. For algorithmic details see [43]. Since ConfJump combines jumps with the hybrid Monte Carlo method, it is not only a correct sampling method for the Boltzmann distribution, but also provides dynamical information.

\subsection{Stability analysis}

In the preceding Section 1.1 we arranged the basic tools for conformation dynamics. On this basis we are now ready to compare the condition of global versus local density computation in the presence of metastable conformations, which is the case of interest here.

Ill-conditioned global density computation. If we want to compute the probabilities for the system to stay within each of the conformations, then we have to solve two problems. The first one is the clustering problem to find the hidden block structure of a transition matrix $P$ and thus to determine the metastable conformations. This problem can be solved by ZIBgridfree and 
$\mathrm{PCCA}+$ as described in the preceding section. The second problem is the determination of the stationary density $\mathbf{w}$ required to compute the discrete cluster weights $\left\{\sigma_{i}\right\}_{i=1}^{n_{C}}$ as defined in (3). Unfortunately, a careful analysis reveals that this problem is ill-conditioned. For a deeper understanding, we will briefly recall the line of argument as given in [44].

Assume we want to compute the stationary density via solving the eigenvalue problem (2). In this case, the truncation error of the transition matrix sampling leads to the following estimate,

$$
\left\|\mathbf{w}-\mathbf{w}_{t r}\right\|_{\infty} \leq \kappa\left\|P-P_{t r}\right\|_{\infty},
$$

where $\mathbf{w}_{t r}, P_{t r}$ indicate the result of the algorithm after truncated sampling, and $\mathbf{w}, P$ the true values. As for the choice of condition number, we select $\kappa=\kappa_{M E}$ suggested by Meyer [30], since it can be conveniently estimated via the sorted eigenvalues $\lambda_{1}=1>\lambda_{2} \geq \ldots \geq \lambda_{N}$ of $P$ as

$$
\frac{1}{N\left|1-\lambda_{2}\right|} \leq \kappa_{M E} \leq \frac{2(N-1)}{\Pi_{i=2}^{N}\left(1-\lambda_{i}\right)} .
$$

In view of (9), we thus obtain that, in the presence of at least two conformations,

$$
\kappa \sim \mathcal{O}(1 / \varepsilon) \gg 1
$$

Summarizing, we are now ready to understand the computational difficulties arising in the existing conformation dynamics algorithms:

- In the presence of conformations, when there exists a Perron cluster of eigenvalues of $P$ near the Perron root $\lambda_{1}=1$, i.e. when $\varepsilon \ll 1$, the computation of the global stationary density $\mathbf{w}$ is ill-conditioned.

- If the second eigenvalue $\lambda_{2}$ of $P$ is sufficiently bounded away from 1 , i.e. if $\varepsilon$ is not small, then the computation of the stationary density is wellconditioned, but PCCA + will not identify any metastable conformations.

The theoretical results so far can also be summarized in a slightly different wording as follows. While the absolute error $\left\|P-P_{t r}\right\|_{\infty}$ can be controlled by the stopping criterion within ZIBgridfree, the element-wise relative error

$$
\left|P(i, j)-P_{t r}(i, j)\right| /|P(i, j)|
$$

is important for the determination of the stationary density $\mathbf{w}$ in the presence of at least two conformations. If the transition probability between two states is small, then the relative error may be very large although the sampling converged and the absolute error is moderate. In this case $\kappa$ in equation (10) will necessarily be large.

Well-conditioned local density computation. Assume there is a molecular system with $n_{C}$ pronounced metastable conformations. Then there exists a Perron cluster of eigenvalues $\lambda_{1}, \ldots, \lambda_{n_{C}}$ and an eigenvalue $\lambda_{n_{C}+1}$ sufficiently bounded away from $\lambda_{n_{C}}<1$. Via PCCA + we can identify the corresponding hidden block-structure of $P$. Assume we have identified disjoint index subsets $I_{1}, \ldots, I_{n_{C}}$ for each of these hidden blocks and reordered the states accordingly 
(what we do not do explicitly!). Then we can construct a real block-diagonal matrix $\hat{P}$ similar to $P$ by applying the Simon-Ando disaggregation technique [39]. That means, we take $P$, set all outer-block elements equal to 0 , which is a perturbation of order $\epsilon$, and change the diagonal elements such that $\hat{P}$ is stochastic again. This matrix $\hat{P}$ is reducible and can be split into $n_{C}$ stochastic sub-matrices $\left\{P_{i}\right\}$. An easy calculation shows that each of these sub-matrices defines a reversible Markov chain with a stationary density equal to the corresponding part $\left\{\mathbf{w}_{I_{i}}\right\}_{i=1}^{n_{C}}$ of $\mathbf{w}$ apart from a scale factor. Due to reversibility, $D P$ and $D \hat{P}$ are symmetric. A standard eigenvalue estimate [19] then yields that $\lambda_{n_{C}+1}$ is a measure for the deviation of the second largest eigenvalue of each sub-matrix from 1 . Since, by assumption, $\lambda_{n_{C}+1}$ is well bounded away from 1 , the upper bound in (11) yields a condition number $\hat{\kappa}_{i}$ for each of the sub-matrices such that

$$
\hat{\kappa}_{i} \ll \kappa,
$$

i.e. local density computation is well-conditioned. This result strongly indicates that some aggregation/disaggregation technique might be useful. This idea will be worked out in Section 2.

\subsection{An illustrative example}

For an illustration of the above stability analysis, consider a two-dimensional dynamical system given by Hamiltonian differential equations,

$$
\dot{q}=p, \quad \dot{p}=-\nabla V(q) .
$$

Let be given a characteristic potential energy $V: \mathbb{R}^{2} \rightarrow \mathbb{R}$ in the form

$$
\begin{gathered}
\qquad(q)=\min (f(q), g(q), h(q)), \quad q \in \mathbb{R}^{2} \\
f(q)=3\left(q_{1}-3\right)\left(q_{1}-3\right)-5\left(q_{1}-3\right)\left(q_{2}+4\right)+3\left(q_{2}+4\right)\left(q_{2}+4\right)+0.25 \\
g(q)=3\left(4-q_{1}\right)\left(4-q_{1}\right)-5\left(4-q_{1}\right)\left(q_{2}-3\right)+3\left(q_{2}-3\right)\left(q_{2}-3\right)+0.25 \\
h(q)=3 q_{1} q_{1}+3 q_{2} q_{2}
\end{gathered}
$$

This potential has three well-separated local minima. The central minimum has the deepest potential energy, but at the same time the smallest cluster weight such that it is impossible to derive the cluster weights from the energy values of the local minima. The potential gives rise to a Boltzmann density with well-separated partial densities, see Figure 2(a). Two of the conformations are symmetric, but their basins of attraction have different orientations, which is disadvantageous for the original ConfJump method [43]. The weights of the three conformations can be computed analytically (rounded to four decimal digits) as

$$
\left\{\sigma_{i}^{\text {exact }}\right\}=\{0.3690,0.2619,0.3690\} .
$$

Application of ZIBgridfree. Since the single peaks are completely separated and transitions from one cluster to another are rare events, we cannot expect ZIBgridfree to deliver the correct stationary density.

We used $N=11$ basis functions $\left\{\phi_{k}\right\}_{k=1}^{N}$. The two symmetric minima were covered by different numbers of basis functions (three and four) so that the 


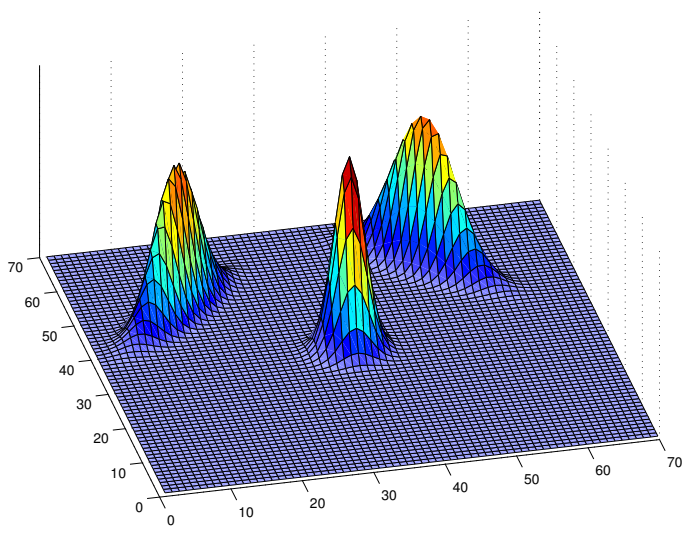

(a) Correct density.

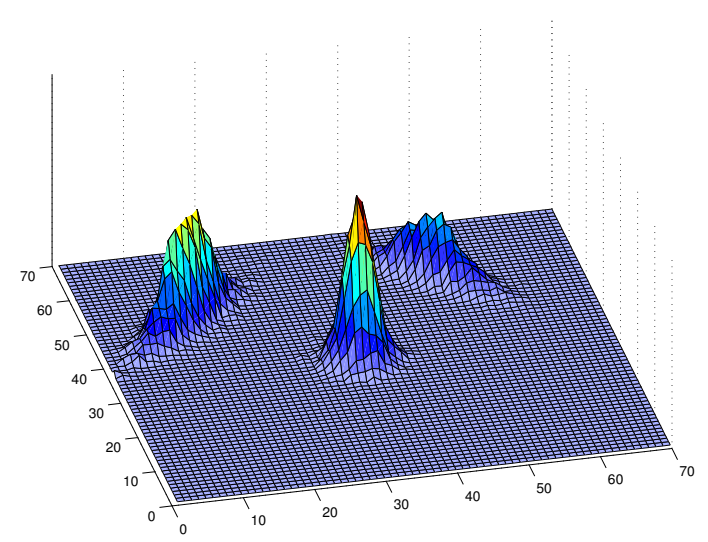

(b) Approximate density computed as left eigenvector of the transition probability matrix.

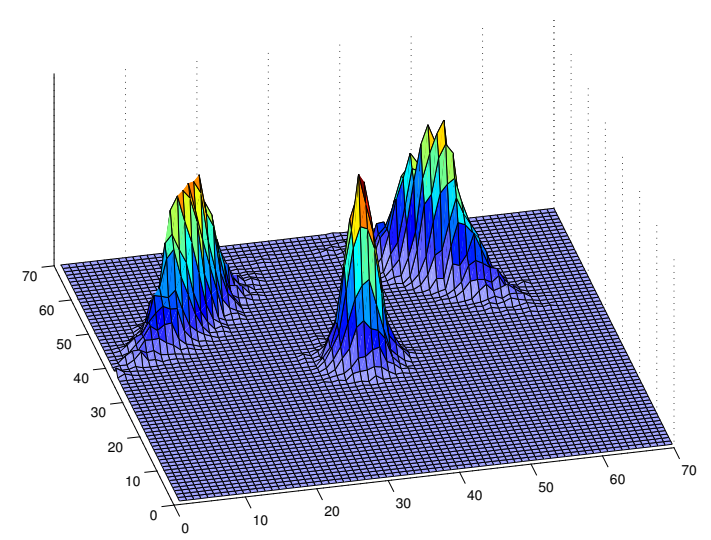

(c) Approximate density computed by ConfJump.

Figure 2: Artificial Potential: Spatial Boltzmann density computed in three different ways. 
discretization does not inherit the symmetry. Moreover, the minima directly coincide with the center of one of the basis functions. The central minimum is resolved by four symmetrically located basis functions. As a result of our algorithm, the basis functions should have the same statistical weights.

For each modified potential $\left\{V_{i}\right\}_{i=1}^{N}$ as defined in (7), 2000 points were generated by hybrid Monte-Carlo sampling. These points were propagated by Hamiltonian dynamics applying 60 steps of the velocity-Verlet algorithm [16] with time step $\Delta \tau=0.013$ fs. We applied PCCA + to the $11 \times 11$ transition probability matrix $P$ which resulted in a decomposition into three clusters.

Ill-conditioned global density computation. The stationary density was calculated by solving the eigenvector problem of $P^{\top}$ according to the eigenvalue $\lambda=1$. The eigenvector was computed by the Matlab routine eigs [18] based on the implicitly restarted Arnoldi method. The Meyer condition number for this calculation appeared as

$$
\kappa=2 \cdot 10^{11}
$$

Well-conditioned local density computation. On the basis of the clustering, we approximated the transition matrix $P$ by a block-diagonal matrix $\hat{P}$ in that we added the entries outside the blocks row-wise to the diagonal, a process described in Section (1.2) above. Next, we calculated the stationary densities of the blocks. This time, the corresponding Meyer condition numbers arose as

$$
\left\{\hat{\kappa}_{i}\right\}=\{2.15,4.10,4.69\}
$$

As one can see, the local condition numbers are extremely smaller than the global condition number.

Computation of cluster weights. We decomposed the density according to the proposed clustering into the partial densities. We know that these partial densities are reliable because our samplings were rapidly mixing within the clusters. But we do not know the ratios or proportionality factors exactly. The cluster weights computed according to (3) came out wrong as

$$
\left\{\sigma_{i}\right\}=\{0.4303,0.3453,0.2244\}
$$

Given the statistical weights $\left\{w_{i}\right\}_{i=1}^{n_{C}}$ of the basis functions, we computed a histogram of the Boltzmann density. For this purpose, we decomposed $\Omega$ into $70 \times 70$ boxes. Then we counted the number of sampling points within each of these boxes. In this counting process, every point $q$ was scaled by a factor

$$
\mu(q)=w_{i}(q) / M_{i}
$$

where $w_{i}(q)$ is the weight of the basis function $i$ from which $q$ was generated, and $M_{i}$ is the total number of points that was generated to sample the Boltzmann distribution according to basis function $i$. The histogram according to our example is illustrated in Figure 2(b).

The computed weights are expected to reflect the symmetry of the potential energy surface. Obviously, this is not true in Figure 2(b). Hence, these weights must be incorrect - in agreement with the analysis in Section 1.2 above. 


\section{Sampling based aggregation/disaggregation}

Recall from Section 1.2 that the stationary density $\mathbf{w}$ can, in principle, be computed as left eigenvector of the stochastic matrix $P$ to the Perron eigenvalue $\lambda=1$. In the presence of at least two metastable conformations, this eigenvector is very close to further eigenvalues in the Perron cluster. The computation of the degenerate Perron cluster eigenspace as a whole is well-conditioned, but the computation of any eigenvector in this space is ill-conditioned. This is the situation, when the matrix $P$, which has been computed by truncated sampling, is given as the input and the stationary density $\mathbf{w}$ to be computed is the output. The result of this insight is that the computation of the matrix as such introduces some algorithmic instability.

In order to circumvent this instability, we will step back behind the computation of $P$, i.e. we will modify the underlying sampling technique. Therefore, in the present section, we will replace the global sampling, which gives rise to the instability introduced via the ill-conditioned eigenvalue problem for the matrix $P$, by a set of independent local samplings corresponding to sub-matrices $\left\{P_{i}\right\}$ associated with well-conditioned eigenvalue problems for the computation of sufficiently accurate partial (or local) densities.

\subsection{Basic idea}

The key idea to overcome the above described computational difficulties is to transfer the well-known matrix based aggregation/disaggregation approach due to Simon and Ando [39] to some domain decomposition realized in the frame of the sampling technique.

Matrix based aggregation/disaggregation. The computation of stationary densities of Markov chains has been the topic of intensive research for many years $[32,40,9,3]$. Iterative aggregation/disaggregation (IAD) methods as introduced in $[26,5,25,41]$ turned out to be efficient algorithms for nearly completely decomposable Markov chains. IAD methods are matrix based domain decomposition methods. Here, domain decomposition refers to the sampling space, and the states of the Markov chain are defined by the partitioning $\left\{\Omega_{i}\right\}$. First, in the disaggregation step, the states $\Omega_{i}$ are grouped to $n_{C}$ subsystems (in the following called clusters) which are solved separately. Then, in the aggregation step, the global solution is constructed from the partial solutions. In other words, the unknown overall vector $\mathbf{w}$ is divided into partial vectors $\left\{\mathbf{w}_{i}\right\}_{i=1}^{n_{C}}$ which may well be of different dimensions,

$$
\mathbf{w}=\left(\mathbf{w}_{1}, \ldots, \mathbf{w}_{n_{C}}\right), \quad\|\mathbf{w}\|_{1}=1 .
$$

The solutions of the disaggregation step are the partial densities

$$
\widetilde{\mathbf{w}}_{i}=\frac{\mathbf{w}_{i}}{\left\|\mathbf{w}_{i}\right\|_{1}}
$$

where the overall normalization constants,

$$
\widetilde{\sigma}_{i}:=\left\|\mathbf{w}_{i}\right\|_{1}, \quad i=1, \ldots, n_{C}
$$

are unknown. These normalization constants denote the discrete cluster weights. Note that they are special instances of $\left\{\sigma_{i}\right\}$ defined in (3) in the case that $\left\{\chi_{i}\right\}$ 
are characteristic functions. From a coupling matrix one can estimate these missing cluster weights which are then used to construct the overall density in the aggregation step,

$$
\mathbf{w}=\left(\widetilde{\sigma}_{i} \widetilde{\mathbf{w}}_{i}, \ldots, \widetilde{\sigma}_{n_{C}} \widetilde{\mathbf{w}}_{n_{C}}\right) .
$$

The method benefits from the fact that the computation of the partial vectors $\mathbf{w}_{i}$ is well-conditioned. An iterative process, which alternately estimates $\left\{\widetilde{\sigma}_{i}\right\}$ and $\mathbf{w}$, accounts for the interactions between the subsystems. These interactions are given by transition probabilities between states which belong to different clusters. If these values were correct, the IAD algorithm would compute the stationary density efficiently. However, our problem is different in that we cannot rely on these values. That means, we cannot apply the method directly to our transition matrix because the aggregation step would fail. Consequently, we had to find a method for the computation of the cluster weights which does not rely on the matrix.

Transfer to sampling techniques. There exist several sampling methods which are based on aggregation/disaggregation techniques. One of them is the hierarchical uncoupling-coupling Monte Carlo method (UCMC) by Fischer $[15,14]$. To approximate ratios of normalizing constants of different densities, UCMC uses temperature based bridge sampling. However, that technique suffers from the fact that the densities must be sampled correctly on every hierarchy level which is very expensive. In our case, here configuration based bridge sampling [28, 17] would also fail due to missing data in the overlap of sampling regions and due to different shapes of these regions.

This situation motivated the development of a new approach which does not suffer from drawbacks as described above. The partitioning of the molecular state space into $N$ different sampling regions as in ZIBgridfree can be considered as the disaggregation step. It also includes the clustering of states into metastable conformations. This partitioning is based on the eigenvector structure of the transition matrix $P$ which is analyzed via PCCA $+[12]$. With the result of the clustering we obtain normalized partial densities

$$
\tilde{\pi}_{i}(q)=\frac{\chi_{i}(q) \pi(q)}{\sigma_{i}}, \quad i=1, \ldots, n_{C}
$$

This definition is comparable to the definition of local densities in (6), but here partial densities correspond to clusters whereas local densities correspond to the discretization. In order to construct the global density

$$
\pi(q)=\sum_{i=1}^{n_{C}} \sigma_{i} \widetilde{\pi}_{i}(q)
$$

one has to estimate the normalizing constants $\left\{\sigma_{i}\right\}_{i=1}^{n_{C}}$ which equal the weights of the conformations. This is part of the sampling based aggregation step which will be described next.

Algorithmic structure. The relation of the different parts of the algorithm together with the stability aspects is schematically presented in Figure 3. The sampling based aggregation/disaggregation algorithm carefully observes the condition of the global versus the local eigenvalue problems as analyzed in Section 


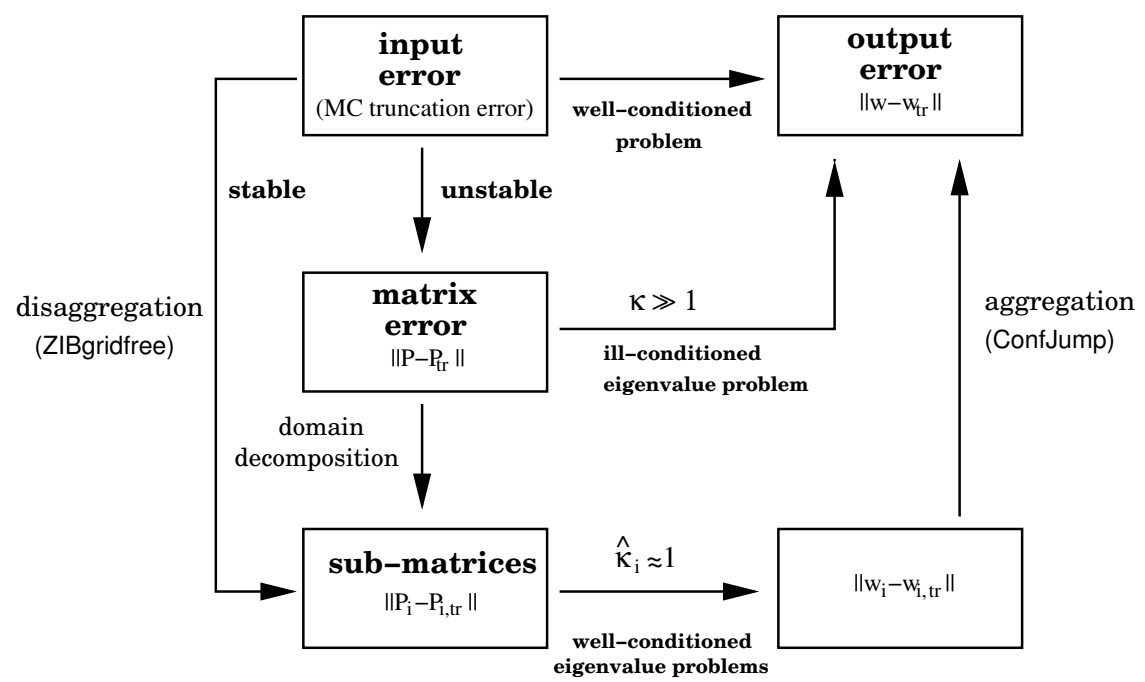

Figure 3: Stability diagram corresponding to basic aggregation/disaggregation algorithm.

1.2 above. Note that in passing we thus have assured that the overall problem as such is well-conditioned. The questions of how to realize this basic algorithm in detail will be discussed in the forthcoming sections.

\subsection{Global aggregation}

Assume we are given the correct partial densities apart from the scaling factors. ConfJump can generate sampling points according to the partial Boltzmann densities even if they are nearly completely separated. Simply counting the points results in the correct scaling of the partial densities. Since we want to avoid re-sampling within conformations, they are replaced by small patches, in our case "domains" of selected basis functions. By applying ConfJump to these "domains", we are able to weight them against each other and thus to compute the correct cluster weights for all conformations. For each conformation we

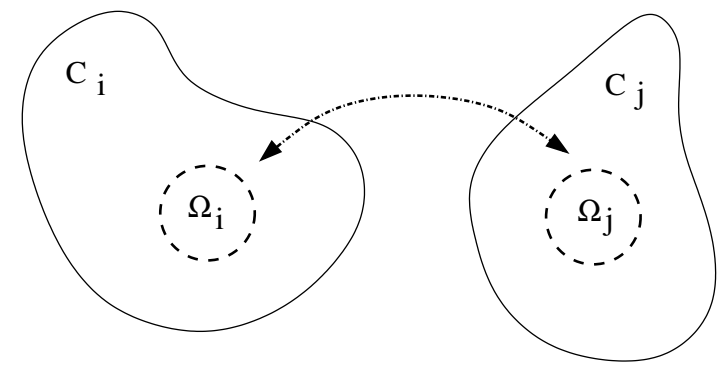

Figure 4: Application of ConfJump to selected "domains" $\Omega_{i} \subset C_{i}, \Omega_{j} \subset C_{j}$ corresponding to basis functions $\phi_{i}, \phi_{j}$. 
select the basis function with maximum weight within this cluster. From the original $N$ basis functions, we only have $n_{C}$ patches at the start of ConfJump, see Figure 4. After renumbering the indices, denote these basis functions by $\phi_{1}, \ldots, \phi_{n_{C}}$ and the statistical weights of them, computed with ZIBgridfree, by $w_{1}, \ldots, w_{n_{C}}$. Recall that the computation of these weights is ill-conditioned and the goal of ConfJump here is to correct these weights. For each basis function $\phi_{i}$ we select a representative node $m_{i}$, for example the point of minimum energy from the trajectory that was generated to sample the Boltzmann distribution according to $V_{i}$. The jump proposition matrix $A$ is computed as described in [43]. One step of the adapted ConfJump method reads as follows:

1. Let $q$ be the initial configuration (in Cartesian coordinates) w.r.t. basis function $\phi_{i}$

2. Pick a random number $z$ in $(0,1)$.

3. If $z>$ jumpRate, perform a hybrid Monte Carlo step within basis function $\phi_{i}$ (i.e. with the modified potential $V_{i}$ )

4. Else, perform a jump step :

(a) Select another basis function $\phi_{j}$ with probability $A_{i j}$.

(b) Compute $x$, the internal coordinates (Z matrix) of $q$.

(c) Compute $\tilde{x}=x+\left(m_{j}-m_{i}\right)$, and transform $\tilde{x}$ to Cartesian coordinates to get the new point $\tilde{q}$.

(d) Accept the new point with probability $\min \left(1, e^{-\beta\left(V_{j}(\tilde{q})-V_{i}(q)\right)}\right)$ (Metropolis acceptance criterion with modified potentials).

The numbers of sampled points within each of the patches divided by the number of all ConfJump sampling points give the new weights $\left\{w_{i}^{\text {new }}\right\}_{i=1}^{n_{C}}$. Thus we get correction factors

$$
f_{j}=\frac{w_{j}^{\text {new }}}{w_{j}}, \quad j=1, \ldots, n_{C} .
$$

Based on the assumption that the ratios $w_{i} / w_{k}$ are correct if basis functions $i$ and $k$ belong to the same cluster, we consider the factors $f_{j}$ as correction factors for all statistical weights of basis functions belonging to conformation $j$. This assumption is correct if the local weight computation is well-conditioned. Now we need to multiply the $N$ original statistical weights by the correction factor corresponding to their conformation. A crisp clustering $\bar{\chi}$ can be performed on the basis of the membership functions $\chi$. The entry $\bar{\chi}(i, j)$ is set to 1 if cluster $j$ is the one to which basis function $i$ belongs with largest probability, and 0 otherwise. Given the crisp membership vectors $\bar{\chi}$, the new statistical weights $\left\{w_{i}^{\text {new }}\right\}_{i=1}^{N}$ of the basis functions can be computed as

$$
w_{i}^{\text {new }}=\sum_{j=1}^{n_{C}} \bar{\chi}(i, j) w_{i} f_{j}, \quad i=1, \ldots, N .
$$

After normalization we obtain the corrected statistical weights of each basis function. This leads to the corrected cluster weights of the conformations via $(3)$. 
Note that theoretically the correction factors can be determined as accurately as needed if the ConfJump sampling has run long enough. As an advantage, the success of ConfJump does not depend on any local regularity of the potential energy function, which is a key feature of Monte-Carlo-based algorithms. Although ConfJump also works without a decomposition method like ZIBgridfree, it is only applicable for larger molecules in combination with ZIBgridfree. This is due to the fact that larger molecules have basins of attraction with very different shapes and energies which leads to low acceptance ratios in the jump algorithm $[43,33]$. Only jumps near the position states $m_{i}$ have a higher probability to be accepted. Therefore, ConfJump benefits from the restriction to smaller regions via ZIBgridfree.

\subsection{Local aggregation via density estimation}

Sometimes the acceptance ratio in the ConfJump algorithm is undesirably low, especially if the jump patches have large differences in shape and in the average energy. In such cases, an alternative aggregation method can be used, known as density estimation. This heuristical method does not use an iterative sampling strategy. Thus, there is no convergence against the correct re-weighting factors. Density estimation constructs an estimate for the underlying continuous Boltzmann density function based on the observed sampling points. Given a sample $\left\{q_{k}\right\}_{k=1}^{n}$ of points in $\Omega$, the numerically computed density at an arbitrary point $q$ can be estimated by the kernel density approximation [31]

$$
\hat{\pi}(q)=\frac{1}{n} \sum_{k=1}^{n} W\left(q-q_{k}\right) .
$$

As kernel function $W$, we usually choose an exponential function

$$
W(q)=\exp (-c\|q\|), \quad c \in \mathbb{R},
$$

which is similar to the basis functions used within ZIBgridfree. In our case, the points $\left\{q_{k}\right\}$ were sampled according to $N$ different basis functions. The points $\left\{q_{k}^{(l)}\right\}$ correspond to the basis function $\phi_{l}$. With the statistical weights $\left\{w_{l}\right\}_{l=1}^{N}$ of these basis functions computed by $\mathrm{PCCA}+$, the formula for the density estimation must be modified in the following way,

$$
\hat{\pi}(q)=\frac{1}{N} \sum_{l=1}^{N} \frac{w_{l}}{M_{l}} \sum_{k=1}^{M_{l}} W\left(q-q_{k}^{(l)}\right),
$$

where $M_{l}$ denotes the number of sampling points corresponding to basis function $l$.

Furthermore, we know the unnormalized value of the restricted Boltzmann density at an arbitrary point $q$,

$$
\pi(q) \propto \exp (-\beta V(q)) .
$$

This allows us to compute correction factors. As in the previous section, we first select the basis function with maximum weight within each cluster. Then we choose from the sampling points corresponding to this basis function the point 
with minimum energy. For these points $\left\{m_{j}\right\}_{j=1}^{n_{C}}$ we compute the estimated densities $\hat{\pi}\left(m_{j}\right)$ according to (15) and scale them such that $\sum_{j} \hat{\pi}\left(m_{j}\right)=1$. Moreover, we calculate $\tilde{\pi}\left(m_{j}\right)=\exp \left(-\beta V\left(m_{j}\right)\right) / \sum_{j} \exp \left(-\beta V\left(m_{j}\right)\right)$. The ratios

$$
f_{j}=\tilde{\pi}\left(m_{j}\right) / \hat{\pi}\left(m_{j}\right), \quad j=1, \ldots, n_{C}
$$

are the correction factors for the weights of cluster $j$. Now we multiply the $N$ original statistical weights of the basis functions by the correction factor corresponding to their conformation, as shown in equation (13). Then we apply

(3) and normalize to obtain the cluster weights.

Since this method does not need a new sampling, it is very fast and cheap. One might have the impression that the approach via local density estimation outperforms the ConfJump algorithm. However, one should be aware that local density estimation only works well in areas where the potential energy surface is sufficiently smooth. This is especially satisfied for small systems and near minima. As already mentioned, ConfJump does not depend on any local regularity assumption.

\section{$2.4 \quad$ Numerical Examples}

The advantage of the aggregation/disaggregation methods will become clear in the following examples. We shortly revisit the artificial example from Section 1.3 and then present two biochemical applications.

Artificial potential. Recall Section 1.3 where the stationary density had been computed as eigenvector of the transition probability matrix, which meant that the cluster weights were incorrect.

This is the point where ConfJump comes into play. Within each cluster, we select the basis function with largest weight and apply the jump method to obtain the scaling factors of the partial densities $\left\{\tilde{\pi}_{i}(a)\right\}$. Then we obtain the corrected cluster weights,

$$
\left\{\sigma_{i}\right\} \approx\{0.3777,0.2585,0.3637\}
$$

to be compared with $\left\{\sigma_{i}^{\text {exact }}\right\}$ in $(12)$. The corresponding histogram is illustrated in Figure 2(c). Now it reflects the symmetry of the potential energy quite satisfactorily and the approximated cluster weights agree with the analytical weights $\sigma_{i}^{\text {exact }}$. Alternatively, we corrected the height of the histogram by a local density approximation. We used the exponential kernel (14) with $c=6.5$. Other values for $c$ in the range $[4,10]$ were tested as well but the results were insensitive w.r.t. the choice of this constant. We obtained the following cluster weights:

$$
\left\{\sigma_{i}\right\} \approx\{0.3798,0.2611,0.3591\} .
$$

The weights agree quite well with the cluster weights obtained by ConfJump and the exact weights in (12).

Bicyclononane (BCN). This molecule is a hydrocarbon which consists of two connected 6-rings. Each of these rings has two major conformations, the chair (C) and the boat (B) conformation. Thus, we expected four possible combinations $(\mathrm{CC}),(\mathrm{CB}),(\mathrm{BC})$, and $(\mathrm{BB})$, all separated by high energy barriers. A 
computation with ZIBgridfree at a temperature of $300 \mathrm{~K}$ with eight basis functions $\phi_{1}, \ldots, \phi_{8}$ led to an $8 \times 8$-transition matrix $P$ with the following spectrum:

$$
\left\{\lambda_{i}\right\}=\{1.000,0.999,0.986,0.972,0.916,0.848,0.774,0.692\} .
$$

We have $\epsilon=1-\lambda_{2}=0.001$ and a significant gap after $\lambda_{4}$. Hence we apply PCCA + with $n_{C}=4$. The uncorrected cluster weights of the four conformations $(\mathrm{CC}),(\mathrm{CB}),(\mathrm{BC})$, and $(\mathrm{BB})$ came out to be:

$$
\left\{\sigma_{i}\right\} \approx\left\{0.943,0.056,0.001,2 \cdot 10^{-14}\right\}
$$

Obviously, the expected symmetry of the (CB) and the (BC) conformation does not show up correctly. The reason for that is, that due to

$$
\kappa \sim 1 / \epsilon \approx 1000
$$

the global weight computation is not reliable. Note that a correct weight computation does not only depend on $\kappa$, but also on the expected truncation error $\left\|P-P_{t r}\right\|_{\infty}$. A variance-based estimation provides the truncation error $\left\|P-P_{t r}\right\| \approx 0.008$. Unfortunately, if we want to reduce this error by a factor of 10 , we have to sample a factor of 100 times more points. As described in Section 1.2 , we computed the transition probability matrix $\hat{P}$ corresponding to a completely decoupled Markov chain. The maximum condition number $\hat{\kappa}$ for the sub-matrices of $\hat{P}$ is

$$
\hat{\kappa}=\max _{i} \hat{\kappa}_{i}=6.17
$$

In order to scale the well-sampled local densities against each other, we applied the pointwise density estimation for a correction of the weights. Here, in contrast to the artifical example, $V(q)$ is invariant against rotation and translation of $q \in \Omega$. Therefore, we first aligned the trajectories to the points where we wanted to estimate the density via Kabsch's alignment algorithm [23, 24]. Via local density estimation (Section 2.3) we computed the corrected cluster weights of the four conformations as

$$
\left\{\sigma_{i}\right\} \approx\left\{0.9893,0.0053,0.0054,3 \cdot 10^{-16}\right\}
$$

In both examples we used an irregular decomposition of the conformational space into basis functions $\phi_{1}, \ldots, \phi_{N}$. The discretization did not reflect the symmetry of the molecule. This means that the reproduction of the molecular symmetry within the weight computation is not an artificial result but a generic property of the described algorithms and can be viewed as a proof of concept.

Inhibitor BSI. The molecule BSI (short name of 2-(Biphenyl-4-sulfonyl)1,2,3,4-tetrahydro-isoquinoline-3-carboxylate) is a possible inhibitor for human neutrophil collagenase (MMP-8), which degrades collagen.

Not only the cluster weights $\sigma_{i}$ inherit the symmetry of the molecule: If a change in internal degrees of freedom of the molecule leads to chemically identical structures, all corresponding histograms for these degrees of freedom are symmetric, too. BSI is an example of a molecule for which many sampling steps are necessary in order to compute the correct probability densities. Figure 5 shows this molecule as structural formula. 


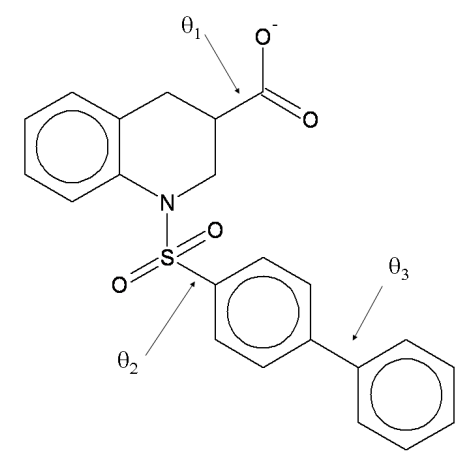

Figure 5: Possible MMP-8 inhibitor BSI with three torsion angles $\theta_{1}, \theta-2$, and $\theta_{3}$ which induce symmetric conformational changes. The values of $\theta_{1}$ are symmetrically distributed, because the negative charged COO-group has a delocated electron pair instead of a single and a double (C-O)-bond.

BSI has three rotationally symmetric bonds. We discretized the configuration space of BSI into 187 basis functions $\phi_{1}, \ldots, \phi_{187}$. We performed a sampling with 3000 up to 15000 steps per basis function (total number of steps: $\approx 1$ million), depending on a convergence indicator. We propagated each sampling point by a molecular dynamics simulation with randomized initial momenta for a time-span $\tau=78$ fs. After computation of the $187 \times 187$-transition matrix $P$ we computed the ill-conditioned, incorrect statistical weights $\left\{w_{i}\right\}_{i=1}^{187}$ of the basis functions.

In Figure 6(a) the results for the pure ZIBgridfree approach are plotted with dashed lines. The histograms do not reflect the correct symmetry of the three bonds. But after local density estimation as in Section 2.3 we corrected the statistical weights and found that the histograms (dash-dotted line in Figure 6(b)) are symmetric now. However, note that for this special example the good results of the local density estimation are due to the symmetric choice of the center points $q$ for the kernel density approximation. In the example of BSI, each of the 187 basis functions was used for the pointwise density estimation. If the re-weighting process with ConfJump is based on each single basis function, this is comparable to performing the pure ConfJump method for the original potential. In the case of BSI, the pure ConfJump approach with 1 million sampling points leads to very good dihedral histograms [33], see Figure 6(c). It was possible for the first time to sample the Boltzmann density in such a way that it correctly reflects the inherent symmetry of the molecule. The jump acceptance ratio was about $32 \%$, which is very high for this approach [43]. We repeated the ConfJump approach with different random number sequences and found out that it is very stable.

Knowing a good approximation of the stationary density, we can compute the coupling matrix $\mathcal{P}$ according to (4). We obtained two conformations with

$$
\mathcal{P}=\left(\begin{array}{ll}
0.999987 & 0.000013 \\
0.000002 & 0.999998
\end{array}\right) .
$$

They result from a rotation of the unsymmetric N-S-bond. With time span 

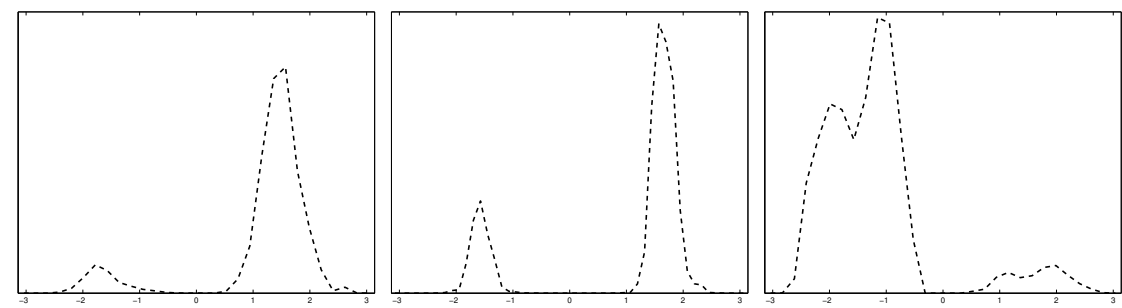

(a) ZIBgridfree without re-weighting. The lack of symmetry clearly indicates that the statistical weights of the discretized states are incorrect.
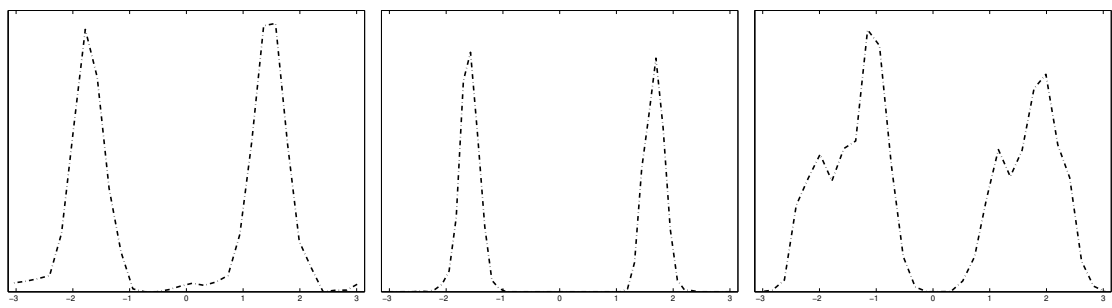

(b) Same sampling as in Figure 6(a), but re-weighted by pointwise density estimation. Here, symmetry is nicely reproduced.
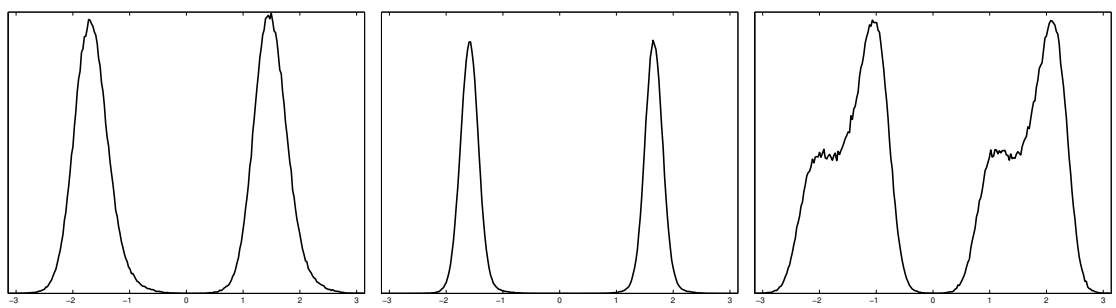

(c) ConfJump re-weights the sampling points in such a way that they correctly represent the Boltzmann distribution. Here, histograms came out symmetric.

Figure 6: Inhibitor BSI: Equilibrium distribution of values for the dihedral angles $\theta_{1}, \theta_{2}$, and $\theta_{3}$ (from left to right) obtained from different samplings of the Boltzmann density (from top to bottom). Symmetry of the histograms in the interval $[-\pi, \pi]$ w.r.t. zero indicates that the Boltzmann density was sampled correctly. 
$\tau=0.78 \mathrm{fs}$, the life times computed according to (5) are

$$
\tau_{1} \doteq 6 \mathrm{~ns}, \quad \tau_{2} \doteq 39 \mathrm{~ns} .
$$

These numbers demonstrate how our model can be used to predict the long-term behavior of molecules from short-term molecular simulations. Thus we are able to bridge the time-gap between molecular simulations, which use time steps of femtoseconds, and chemical experiments in the range of nanoseconds.

\section{Conclusion}

The paper suggests a stable aggregation/disaggregation algorithm for the wellconditioned computation of stationary densities of metastable dynamical systems. The algorithm as it stands now scales with the number of identified conformations and can be used as a postprocessor applied to traditional conformation analysis. Future work will aim at telescoping the traditional tools with this postprocessor.

\section{References}

[1] Amira-advanced visualization, data analysis and geometry reconstruction, user's guide and reference manual. Konrad-Zuse-Zentrum für Informationstechnik Berlin (ZIB), Indeed-Visual Concepts GmbH and TGS Template Graphics Software Inc., 2000.

[2] I. Andricioaei, J. Straub, and A. Voter. Smart darting Monte Carlo. J. Chem. Phys., 114(16):6994-7000, 2001.

[3] M. Benzi and M. Tůma. A parallel solver for large scale Markov chains. Appl. Num. Math., 41(1):135-153, April 2002.

[4] B. Berne and J. Straub. Novel methods of sampling phase space in the simulation of biological systems. Current Opinion in Structural Biology, 7:181-189, 1997.

[5] W.-L. Cao and W. J. Stewart. Iterative aggregation/disaggregation techniques for nearly uncoupled Markov chains. JACM, 32(3):702-719, July 1985.

[6] G. E. Cho and C. D. Meyer. Comparison of perturbation bounds for the stationary distribution of a Markov chain. Lin. Alg. App., 335(1-3):137-150, 2001.

[7] P. J. Courtois. Decomposability; Queueing and Computer System Applications. Academic Press, Orlando, Florida, 1977.

[8] T. Dayar. Permuting Markov chains to nearly completely decomposable form. Technical Report BU-CEIS-9808, Department of Computer Science and Engineering, Bilkent University, August 1998.

[9] T. Dayar and W. J. Stewart. Comparison of partitioning techniques for two-level iterative solvers on large, sparse Markov chains. SIAM J. Sci. Comp., 21(5):1691-1705, 2000.

[10] P. Deufhard. From molecular dynamics to conformational dynamics in drug design. In M. Kirkilionis, S. Krömker, R. Rannacher, and F. Tomi, editors, Trends in Nonlinear Analysis, pages 269-287. Springer, 2003.

[11] P. Deuflhard and C. Schütte. Molecular conformation dynamics and computational drug design. In J. M. Hill and R. Moore, editors, Applied Mathemetics Entering the 21st Century. Proceedings ICIAM 2003, pages 91-119, 2004. invited paper.

[12] P. Deuflhard and M. Weber. Robust Perron Cluster Analysis in Conformation Dynamics. In M. Dellnitz, S. Kirkland, M. Neumann, and C. Schütte, editors, Lin. Alg. App. - Special Issue on Matrices and Mathematical Biology, volume 398C, pages 161-184. Elsevier, 2005.

[13] S. Duane, A. D. Kennedy, B. J. Pendleton, and D. Roweth. Hybrid Monte Carlo. Phys. Lett. B, 195(2):216-222, 1987. 
[14] A. Fischer. An Uncoupling-Coupling Method for Markov Chain Monte Carlo Simulations with an Application to Biomolecules. Doctoral thesis, Freie Universität Berlin, 2003.

[15] A. Fischer, Ch. Schütte, P. Deuflhard, and F. Cordes. Hierarchical uncoupling-coupling of metastable conformations. In T. Schlick and H. H. Gan, editors, Computational Methods for Macromolecules: Challenges and Applications - Proc. of the 3rd Intern. Workshop on Algorithms for Macromolecular Modelling, pages 235-259, Berlin, Heidelberg, New York, 2002. Springer.

[16] D. Frenkel and B. Smit. Understanding Molecular Simulation - From Algorithms to Applications, volume 1 of Computational Science Series. Academic Press, 2002.

[17] A. Gelman and X. Meng. Simulating normalizing constants: From importance sampling to bridge sampling to path sampling. Statistical Science, 13:163-185, 1998.

[18] TheMathWorks Inc. Germany. Matlab(R) 6.5.0, 1994-2005.

[19] G.H. Golub and C.F. van Loan. Matrix Computations. Johns Hopkins University Press, 3rd edition, 1996.

[20] M. Haviv and L. van der Heyden. Perturbation bounds for the stationary propabilities of a finite Markov chain. Adv. Appl. Prob., 16:804-818, 1984.

[21] W. Huisinga. Metastability of Markovian systems: A transfer operator based approach in application to molecular dynamics. Doctoral thesis, Freie Universität Berlin, 2001.

[22] I. Ipsen and C. D. Meyer. Uniform stability of Markov chains. SIAM J. Matrix Anal. Appl., 15:1061-1074, 1994.

[23] W. Kabsch. A solution for the best rotation to relate two sets of vectors. Acta Cryst., A32:922-923, 1976.

[24] W. Kabsch. A discussion of the solution for the best rotation to relate two sets of vectors. Acta Cryst., A34:827-828, 1978.

[25] H. D. Kafeety, C. D. Meyer, and W. J. Stewart. A general framework for iterative aggregation /disaggregation methods. In Proceedings of the Fourth Copper Mountain Conference on Iterative Methods, 1992.

[26] R. Koury, D. F. McAllister, and W. J. Stewart. Iterative methods for computing stationary distributions of nearly completely decomposable Markov chains. SIAM Journal on Algebraic and Discrete Methods, 5(2):164-186, 1984.

[27] S. Kube and M. Weber. Coarse grained molecular kinetics. ZIB-Report 06-35, Zuse Institute Berlin, 2006.

[28] X.-L. Meng and W. H. Wong. Simulating ratios of normalizing constants via a simple identity: A theoretical exploration. Statistica Sinica, 6:831-860, 1996.

[29] C. D. Meyer. Stochastic complementation, uncoupling Markov chains, and the theory of nearly reducible systems. SIAM Rev., 31(2):240-272, 1989.

[30] C. D. Meyer. Sensitivity of the stationary distribution of a Markov chain. SIAM Journal on Matrix Analysis and Applications, 15:715-728, 1994.

[31] E. Parzen. On estimation of a probability density function and mode. Ann. Math. Stat., 33:1065-1076, 1962.

[32] B. Philippe, Y. Saad, and W. J. Stewart. Numerical methods in Markov chain modelling. Operations Research, 40(6), 1992.

[33] A. Riemer. Accuracy, stability, convergence of rigorous thermodynamic sampling methods. Master's thesis, FU Berlin, Bioinformatics, 2006.

[34] J. Schmidt-Ehrenberg, D. Baum, and H.-Ch. Hege. Visualizing dynamic molecular conformations. In IEEE Visualization 2002, pages 235-242. IEEE Computer Society Press, 2002.

[35] Ch. Schütte. Conformational Dynamics: Modelling, Theory, Algorithm, and Application to Biomolecules. Habilitation thesis, Department of Mathematics and Computer Science, Freie Universität Berlin, 1999.

[36] Ch. Schütte and W. Huisinga. Biomolecular Conformations can be Identified as Metastable Sets of Molecular Dynamics. In P. G. Ciarlet and C. Le Bris, editors, Handbook of Numerical Analysis, volume X. Special Volume Computational Chemistry, pages 699-744. North-Holland, 2003. 
[37] H. Senderowitz, F. Guarnieri, and W. C. Still. A smart Monte Carlo technique for free energy simulations of multiconformational molecules. Direct calculations of the conformational populations of organic molecules. J. Am. Chem. Soc., 117:8211-8219, 1995.

[38] H. Senderowitz and W. C. Still. Simple but smart Monte Carlo algorithm for free energy simulations of multiconformational molecules. J. Comp. Chem., 19(15):1736-1745, 1998.

[39] H. A. Simon and A. Ando. Aggregation of variables in dynamical systems. Econometrica, 29(2):111-138, 1961.

[40] W. J. Stewart. Numerical methods for computing stationary distributions of finite irreducible Markov chains. In W. Grassmann, editor, Advances in Computational Probability, chapter 3. Kluwer Academic Publishers, 1997.

[41] W. J. Stewart and W. Wu. Numerical experiments with iteration and aggregation for Markov chains. ORSA J. Comput., 4:336-350, 1992.

[42] G.M. Torrie and J.P. Valleau. Monte Carlo study of a phase-separating liquid mixture by umbrella sampling. J.Chem.Phys., 66(4):1402-1408, February 1977.

[43] L. Walter and M. Weber. ConfJump: a fast biomolecular sampling method which drills tunnels through high mountains. ZIB-Report 06-26, Zuse Institute Berlin, 2006.

[44] M. Weber. Meshless Methods in Conformation Dynamics. Doctoral thesis, Department of Mathematics and Computer Science, Freie Universität Berlin, 2006. published by Verlag Dr. Hut, München.

[45] M. Weber and H. Meyer. ZIBgridfree - adaptive conformation analysis with qualified support of transition states and thermodynamic weights. ZIB-Report 05-17, Zuse Institute Berlin, 2005. 